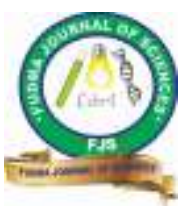

FUDMA Journal of Sciences (FJS)

ISSN online: $2616-1370$

ISSN print: 2645 - 2944

Vol. 4 No. 2, June, 2020, pp 290 - 299

DOI: https://doi.org/10.33003/fjs-2020-0402-219

\title{
CONSTRUCTION AND ANALYSIS OF BALANCED INCOMPLETE SUDOKU SQUARE DESIGN
}

\author{
${ }^{1}$ Dauran, N. S., ${ }^{2}$ Odeyale, A. B. and ${ }^{* 3}$ Shehu, A. \\ ${ }^{1,2}$ Department of Mathematics, Usmanu Danfodiyo University, Sokoto, Nigeria. \\ ${ }^{3}$ Department of Mathematical Sciences, Federal University Dutsin-Ma Katsina State, Nigeria \\ *Corresponding author’s email: ashehu@fudutsinma.edu.ng
}

\begin{abstract}
Sudoku squares have been widely used to design an experiment where each treatment occurs exactly once in each row, column or sub-block. For some experiments, the size of row (or column or sub-block) may be less than the number of treatments. Since not all the treatments can be compared within each block, a new class of designs called balanced incomplete Sudoku squares design (BISSD) is proposed. A general method for constructing BISSD is proposed by an intelligent selection of certain cells from a complete Latin square via orthogonal Sudoku designs. The relative efficiencies of a delete-one-transversal balance incomplete Latin Square (BILS) design with respect to Sudoku design are derived. In addition, linear model, numerical examples and procedure for the analysis of data for BISSD are proposed.
\end{abstract}

Keywords: Balanced incomplete Latin square, Sudoku design, NP-complete.

\section{INTRODUCTION}

A Sudoku design is a block matrix filled with different Latin letters or numbers, such that each letter or number appears in a row (column or sub-block) only once. Hui-Dong and Ru-Gen (2008) stated that all non-prime number can construct a Sudoku square while prime number $k$ cannot, $\mathrm{k}$ is the number of treatments (or rows or columns) of the square. It was presented by Subramani and Ponnuswamy (2009) for the construction of Sudoku designs of order $k=m^{2}$ only and also proposed linear models for analyzing the data obtained from their design, and applied the designs to agricultural experiments. It has been shown that Sudoku design may be incomplete as reported by Bejar et al (1960) and Mahdian Mahmoodian (2015). In addition Donovan et al (2015) and Kumar et al (2015) studied the Sudoku based space filling designs.

Researchers address a classical problem of experimental designs, in the construction of balanced incomplete Latin squares (BILS) design. However the construction of balanced incomplete Sudoku squares design (BISSD) are

popularly known as experimental designs for several reasons; like the randomized complete block designs, balanced incomplete Latin square designs and Latin square designs, they are variance balanced. This problem of constructing balanced incomplete Latin squares has drawn the attention of many leading mathematicians and statisticians for a long period. Recent contribution in this area includes Das and Dey (1990), and Subramani and Ponnuswamy (2009). Where Das and Dey (1990) have presented a simple method of constructing a pair of orthogonal Latin squares of odd order by developing two initial rows. A Graeco Latin square can be obtained by superimposing any two orthogonal Latin squares. By extending the method of
Das and Dey (1990), a more generalized method of constructing several orthogonal (Graeco) Latin squares of odd order to give Latin square was presented by Subramani and Ponnuswamy (2009) . Recently Subramani (2013), extended the work of Das and Dey (1990) and Subramani and Ponnuswamy (2009), to construct and analysis of orthogonal (Graeco) Sudoku square designs. A sequential method of constructing and analyzing orthogonal Sudoku square designs of odd order was presented in the paper.

Mahdian and Mahmoodian (2015) report that a balanced incomplete Sudoku design is an incomplete filled block boxes with some empty cells which satisfied that each letter appears only once in (row, column or sub-block). This balanced incomplete Sudoku design has been shown to be non deterministic polynomial time complete for the particular case of sub-blocks square. In general, Sudoku square is said to be non complete 9complete if a balanced incomplete Sudoku square is completable see Colbourn (1984), and Mahdian and Mahmoodian (2015).

A general method for constructing BILS was discussed by Mingyao et al (2013) for selection of certain cells from a complete Latin square via orthogonal Latin squares. Mingyao et al (2013), investigated the optimality for BILS designs and concluded that the transversal BILS designs are asymptotically optimal for all the row, column and treatment effects. Recently Danbaba et al (2018) presented a paper on joint analysis of several experiments conducted via orthogonal Sudoku square design of odd order and conclude that joint analysis of multi-environment experiments increases the accuracy of evaluation, instead of analyzing each experiment separately. Mingyao et al (2013) also discussed that balanced incomplete Latin square designs occurs only once in 
each row and column of the design. However the analysis in Mingyao et al (2013) is not Sudoku design in nature. This paper proposed construction and analysis of balanced incomplete Sudoku square designs that is to select certain cells from a complete Sudoku square such that the remaining cells satisfy the condition of balanced occurrence of numbers. The method of constructing balanced incomplete Sudoku square designs is presented in section 2 with other related results and numerical examples. Furthermore the computational formulae of the error sum of squares together with the error degree of freedom of four different types of the balanced incomplete Sudoku square designs in line with the four different Sudoku square designs are given in section 3 . Finally the numerical analysis and results are given in section 4 .

Construction of balanced incomplete Sudoku square designs.

A natural way of constructing a BISSD is to select certain cells from a complete Latin square such that the remaining cells satisfy the condition of balanced occurrence of symbols. It can be done by removing one or more "transversal". For a given Latin square $(k)$, a transversal is a set of cells such that only one cell is followed in each row and column, and further, each symbol appears in each cell exactly once. It is known that any two Latin squares of the same order are said to be orthogonal, if these two squares when superimposed have the property that each pair of symbols appears exactly once Bose et al, Subramani and Ponnuswamy (2009), Mingyao et al (2013), Subramani (2013), and Danbaba et al (2018).

Example 1. Consider the experiment from Subramani (2013), for testing the abrasion resistance of rubber-covered fabric in a Martindale wear tester. The original design is the complete Latin square in Table 1(a), where we have LS(4) for four material $\mathrm{A}, \mathrm{B}, \mathrm{C}$ and $\mathrm{D}$ respectively. The response is the loss in weight in $0.1(\mathrm{mg})$ over a period of time. Two blocking variables "application" and "position" are assigned to the rows and columns, respectively. The weight data is given below. Now we consider the BILS $(4,3)$ design obtained in example 1 , the data along the diagonal in Table 1(a) are removed, as shown in Table 1(b). The underlying linear model for BILS $(k, r)$ design is:

$$
Y_{i j l}=\mu+\alpha_{i}+\beta_{j}+\tau_{l}+e_{i j l}
$$

Where $i, j$ takes vales in $(1,2, \ldots, k)$ and $l$ is the symbol in the $(i, j)$-th cell of the $\operatorname{BILS}(k, r), \mu$, is the overall mean, $\alpha_{i}$ is the $i t h$ row effect, $\beta_{j}$ is the $j t h$ column effect, $\tau_{l}$ is the effect of the $l t h$ treatment, and the errors $e_{i j l}$ are the independent $\mathrm{N}\left(0, \sigma^{2}\right)$.

Table 1: Weight loss data for $\operatorname{LS}(4)$ and $\operatorname{BILS}(3)$.

\begin{tabular}{|l|l|l|l|}
\hline $\mathbf{2 3 5}$ & 236 & 218 & 268 \\
\hline 251 & $\mathbf{2 4 1}$ & 227 & 229 \\
\hline 234 & 273 & $\mathbf{2 7 4}$ & 226 \\
\hline 195 & 270 & 230 & $\mathbf{2 2 5}$ \\
\hline
\end{tabular}

\begin{tabular}{|l|l|l|l|}
\hline & 236 & 218 & 268 \\
\hline 251 & & 227 & 229 \\
\hline 234 & 273 & & 226 \\
\hline 195 & 270 & 230 & \\
\hline
\end{tabular}

$\begin{array}{ll}\text { (a) Data of LS(4) } & \text { (b) Data of } \operatorname{BILS}(4,3)\end{array}$

Table 2: Anova table for the BILS $(4,3)$ wear experiment.

\begin{tabular}{|c|c|c|c|c|}
\hline Source & $\begin{array}{l}\text { degree of Sum of } \\
\text { freedom squares }\end{array}$ & $\begin{array}{l}\text { Mean } \\
\text { squares }\end{array}$ & F-value & $\mathrm{P}$-value $(>\mathrm{F})$ \\
\hline Application 3 & 278.2 & 92.75 & 3.66 & 0.22192 \\
\hline Position 3 & 2243.5 & 747.83 & 29.52 & 0.03294 \\
\hline Material & 3424.5 & 1141.50 & 45.06 & 0.02179 \\
\hline Residual 2 & 50.7 & 25.33 & & \\
\hline
\end{tabular}

Balanced incomplete Sudoku squares design.

In this study, four models of orthogonal Sudoku square designs discussed by Subramani (2013) were modified and balanced incomplete Sudoku square from each were discussed under the construction. For all the models considered in this study, it is assumed that all effects are fixed. 
Consider the construction of an incomplete Latin square $\operatorname{ILS}(k, r)$ of order $k$ in which each row and each column has $r$ empty cells. If $\operatorname{ILS}(k, r)$ satisfies the condition that each number appears exactly $(k)$ times in the whole square, then the ILS $(k, r)$ is called a balanced incomplete Sudoku square design.

Example 2. For $k=9$ and $r=3$, let $k-r=x$, suppose we have Latin square of order (3), if we remove the transversal number of Table 3(a) below the cells will be a BILS $(3,2)$ as in Table 3(b) then the balanced incomplete Sudoku square design BISSD(9,6) is obtained as displayed in table 3(c) below.

Table 3: LS(3), BILS(3,2) and BISS(9,6).

\begin{tabular}{|l|l|l|}
\hline $\mathbf{1}$ & 2 & 3 \\
\hline 4 & $\mathbf{5}$ & 6 \\
\hline 7 & 8 & $\mathbf{9}$ \\
\hline
\end{tabular}

(a.) $\operatorname{LS}(3)$

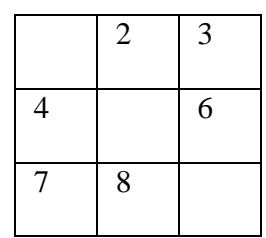

(b) $\operatorname{BILS}(3,2)$

(c) $\operatorname{BISS}(9,6)$

\begin{tabular}{|l|l|l|l|l|l|l|l|l|}
\hline & 2 & 3 & 4 & & 6 & 7 & 8 & \\
\hline 4 & & 6 & 7 & 8 & & & 2 & 3 \\
\hline 7 & 8 & & & 2 & 3 & 4 & & 6 \\
\hline & 6 & 4 & 8 & & 7 & 2 & 3 & \\
\hline 8 & & 7 & 2 & 3 & & & 6 & 4 \\
\hline 2 & 3 & & & 6 & 4 & 8 & & 7 \\
\hline & 7 & 8 & 3 & & 2 & 6 & 4 & \\
\hline 3 & & 2 & 6 & 4 & & & 7 & 8 \\
\hline 6 & 4 & & & 7 & 8 & 3 & & 2 \\
\hline
\end{tabular}

\section{CONSTRUCTION AND ANALYSIS OF BALANCED INCOMPLETE SUDOKU SQUARE DESIGN}

Consider the four models of orthogonal Sudoku square designs discussed by Subramani (2013) below with the error sum of squares together with the corresponding degrees of freedom.

\section{Balanced Incomplete Sudoku square designs - Type 1}

In this model we assume row, column and treatment effects as in the Latin square designs.

In addition to these we assume row block, column block and square as fixed effects model. The Sudoku design of order $(x)$ and its Analysis of Variance (ANOVA) model is given below:

$$
\begin{array}{r}
Y_{i j(l, n, p, q)}=\mu+\alpha_{i x}+\beta_{j x}+\tau_{n}+\gamma_{l x}+C_{p x}+S_{q x}+e_{i j(l, n, p, q)} \\
\text { where } i, j=1,2, \ldots, m, \quad l, n, p, q=1,2, \ldots, x
\end{array}
$$

$\mu=$ General Mean effect

$\alpha_{i, x}=i^{t h}$ Row block effect in $x^{t h}$ empty cells,

$\beta_{j, x}=j^{\text {th }}$ Column block effect in $x^{\text {th }}$ empty cells,

$\tau_{n}=n^{\text {th }}$ Treatment effect

$\gamma_{l, x}=l^{\text {th }}$ Row effect in $x^{\text {th }}$ empty cells,

$C_{p, x}=p^{t h}$ Column effect in $x^{t h}$ empty cells,

$S_{q, x}=q^{t h}$ Square effect in $x^{\text {th }}$ empty cells,

$e_{i j(l, n, p, q)}=$ Error component effect with mean zero and variance $\sigma^{2}$

$G=\sum_{i=1}^{x} \sum_{j=1}^{x} Y_{i j}$ and $N=m^{2} x$

$T S S=\sum_{i=1}^{x} \sum_{j=1}^{x} Y_{i j}^{2}-\frac{G^{2}}{N}$

$S S T=\sum_{n=1}^{x} \frac{\tau_{n}^{2}}{x}-\frac{G^{2}}{N}$ 
$S S R B=\sum_{i=1}^{m} \frac{R B_{i}^{2}}{m x}-\frac{G^{2}}{N}$

$S S C B=\sum_{j=1}^{m} \frac{C B_{i}^{2}}{m x}-\frac{G^{2}}{N}$

$S S R=\sum_{l=1}^{x} \frac{\gamma_{l}^{2}}{x}-\frac{G^{2}}{N}$

$S S C=\sum_{p=1}^{x} \frac{C_{p}^{2}}{x}-\frac{G^{2}}{N}$

$S S S=\sum_{q=1}^{x} \frac{S_{q}^{2}}{x}-\frac{G^{2}}{N}$

$E S S=\sum_{i=1}^{x} \sum_{j=1}^{x} Y_{i j}^{2}-\sum_{i=1}^{m} \frac{R B_{i}^{2}}{m x}-\sum_{j=1}^{m} \frac{C B_{i}^{2}}{m x}-\sum_{n=1}^{x} \frac{\tau_{n}^{2}}{x}-\sum_{l=1}^{x} \frac{\gamma_{l}^{2}}{x}-\sum_{p=1}^{x} \frac{C_{p}^{2}}{x}-\sum_{q=1}^{x} \frac{S_{q}^{2}}{x}+\frac{5 G^{2}}{N}$

Error degrees of freedom $=(m+1)(x-1)$

Table 4. ANOVA table for BISSD Model Type I

\begin{tabular}{|l|l|c|c|c|}
\hline Source & Sum of Squares & Degree of Freedom & Mean Square & F-Ratio \\
\hline Treatments & SST & $x-1$ & $M S T=S S T / d f$ & $M S T / M S E$ \\
\hline Row & SSR & $x-1$ & $M S R=S S R / d f$ & $M S R / M S E$ \\
\hline Column & SSC & $x-1$ & $M S C=S S C / d f$ & $M S C / M S E$ \\
\hline Row-block & SSRB & $m-1$ & $M S R B=S S R B / d f$ & $M S R B / M S E$ \\
\hline Column-block & SSCB & $m-1$ & $M S C B=S S C B / d f$ & $M S C B / M S E$ \\
\hline Sub-Square & SSS & $x-1$ & $M S S=S S S / d f$ & $M S S / M S E$ \\
\hline Error & SSE & $(m+1)(x-1)$ & $M S E=S S E / d f$ & \\
\hline Total & TSS & $m^{2} x-1$ & & \\
\hline
\end{tabular}

Balanced Incomplete Sudoku square designs - Type II

aIn this model it is assumed that the row effects are nested in the row block effects and the column effects are nested in the column block effects. The Sudoku design of order $(x)$ and its Analysis of Variance (ANOVA) model is given below:

$$
\begin{gathered}
Y_{i j(l, n, p, q)}=\mu+\alpha_{i x}+\beta_{j x}+\tau_{n}+\gamma(\alpha)_{l(i) x}+C(\beta)_{p(j) x}+S_{q x}+e_{i j(l, n, p, q)} \\
\text { where } i, j, l, p=1,2, \ldots, m \text { and } n, q=1,2, \ldots, x
\end{gathered}
$$

$\mu=$ General Mean effect

$\alpha_{i x}=i^{\text {th }}$ Block (row) effect in $x^{\text {th }}$ empty cells,

$\gamma_{j x}=j^{\text {th }}$ Block (column) effect in $x^{\text {th }}$ empty cells,

$\tau_{n}=n^{\text {th }}$ Treatment effect

$\gamma(\alpha)_{l(i) x}=l^{t h}$ Row effect nested in ith block (row) in $x^{\text {th }}$ empty cells,

$C(\beta)_{p(j) x}=p^{\text {th }}$ Column effect nested in $j t h$ block (column) in $x^{\text {th }}$ empty cells,

$S_{q x}=q^{t h}$ Square effect in $x^{\text {th }}$ empty cells,

$e_{i j(l, n, p, q)}=$ Error component with mean zero and variance $\sigma^{2}$ 
$T S S=\sum_{i=1}^{m^{2}} \sum_{j=1}^{m^{2}} Y_{i j}^{2}-\frac{G^{2}}{N}$

$S S T=\sum_{n=1}^{x} \frac{T_{n}^{2}}{x}-\frac{G^{2}}{N}$

$\operatorname{SSRB}=\sum_{i=1}^{m} \frac{R B_{i}^{2}}{m x}-\frac{G^{2}}{N}$

$\operatorname{SSCB}=\sum_{j=1}^{m} \frac{C B_{i}^{2}}{m x}-\frac{G^{2}}{N}$

$S S R=\sum_{i=1}^{m} \sum_{l=1}^{m} \frac{R_{l(i)}^{2}}{x}-\sum_{i=1}^{m} \frac{R B_{i}^{2}}{m x}$

$S S C=\sum_{j=1}^{m} \sum_{p=1}^{m} \frac{C_{p(j)}^{2}}{x}-\sum_{j=1}^{m} \frac{C B_{i}^{2}}{m x}$

$S S S=\sum_{q=1}^{m^{2}} \frac{S_{q}^{2}}{x}-\frac{G^{2}}{N}$

$E S S=\sum_{i=1}^{m^{2}} \sum_{j=1}^{m^{2}} Y_{i j}^{2}-\sum_{i=1}^{m} \sum_{l=1}^{m} \frac{R_{l(i)}^{2}}{x}-\sum_{j=1}^{m} \sum_{p=1}^{m} \frac{C_{p(j)}^{2}}{x}-\sum_{n=1}^{x} \frac{T_{n}^{2}}{x}-\sum_{q=1}^{m^{2}} \frac{S_{q}^{2}}{x}+\frac{3 G^{2}}{N}$

Error degrees of freedom $=m(x+2)$

Table 5: ANOVA table for BISSD Model Type II

\begin{tabular}{|l|l|c|c|c|}
\hline Source & Sum of Squares & Degree of Freedom & Mean Square & F-Ratio \\
\hline Treatments & SST & $x-1$ & $M S T=S S T / d f$ & $M S T / M S E$ \\
\hline Row-block & SSRB & $m-1$ & $M S R B=S S R B / d f$ & $M S R B / M S E$ \\
\hline Column-block & SSCB & $m-1$ & $M S C B=S S C B / d f$ & $M S C B / M S E$ \\
\hline Row within RB & SSR(RB) & $m(m-1)$ & $M S R=S S R / d f$ & $M S R / M S E$ \\
\hline Column within CB & SSC(CB) & $m(m-1)$ & $M S C=S S C / d f$ & $M S C / M S E$ \\
\hline Sub-Square & SSS & $x-1$ & $M S S=S S S / d f$ & $M S S / M S E$ \\
\hline Error & SSE & $m(x+2)$ & $M S E=S S E / d f$ & \\
\hline Total & TSS & $m^{2} x-1$ & & \\
\hline
\end{tabular}

Balanced Incomplete Sudoku square designs - Type 1II

In this model it is assumed that the horizontal square effects are nested in the row block effects and the vertical square effects are nested in the column block effects.

$\boldsymbol{Y}_{\boldsymbol{i j}(\boldsymbol{l}, \boldsymbol{n}, \boldsymbol{p}, \boldsymbol{q}, \boldsymbol{w})}=\boldsymbol{\mu}+\alpha_{i x}+\beta_{j x}+\tau_{n}+\gamma_{l x}+C_{p x}+S(\alpha)_{q(i) x}+\pi(\beta)_{w(j) x}+e_{i j(l, n, p, q, w)}$

where $i, j, n, q, w=1, \ldots, m$ and $l, p=1,2, \ldots, x$

$\mu=$ General mean effect

$\alpha_{i x}=i^{\text {th }}$ Row block effect in $x^{\text {th }}$ empty cells,

$\beta_{j x}=j^{\text {th }}$ Column block effect in $x^{\text {th }}$ empty cells,

$\tau_{n}=n^{\text {th }}$ Treatment effect,

$\gamma_{l x}=l^{\text {th }}$ Row effect in $x^{\text {th }}$ empty cells, 
$C_{p x}=p^{t h}$ Column effectin $x^{\text {th }}$ empty cells,

$S(\alpha)_{q(i) x}=q^{\text {th }}$ Horizontal Square effect nested in $i^{\text {th }}$ Row block effect in $x^{\text {th }}$ empty cells,

$\pi(\beta)_{w(j) x}=w^{\text {th }}$ Vertical Square effect nested in $j^{\text {th }}$ Column block effect in $x^{\text {th }}$ empty cells,

$e_{i j(l, n, p, q, w)}=$ Error component with mean zero and variance $\sigma^{2}$

$T S S=\sum_{i=1}^{m^{2}} \sum_{j=1}^{m^{2}} Y_{i j}^{2}-\frac{G^{2}}{N}$

$S S T=\sum_{n=1}^{x} \frac{T_{n}^{2}}{x}-\frac{G^{2}}{N}$

$\operatorname{SSRB}=\sum_{i=1}^{m} \frac{R B_{i}^{2}}{m x}-\frac{G^{2}}{N}$

$\operatorname{SSCB}=\sum_{j=1}^{m} \frac{C B_{i}^{2}}{m x}-\frac{G^{2}}{N}$

$S S R=\sum_{l=1}^{x} \frac{R_{l}^{2}}{x}-\frac{G^{2}}{N}$

$S S C=\sum_{p=1}^{x} \frac{C_{p}^{2}}{x}-\frac{G^{2}}{N}$

$S S H S=\sum_{i=1}^{m} \sum_{p=1}^{m} \frac{S(\alpha)_{q(i)}}{x}-\sum_{i=1}^{m} \frac{R B_{i}^{2}}{m x}$

$S S V S=\sum_{j=1}^{m} \sum_{w=1}^{m} \frac{\pi(\beta)_{w(j)}}{x}-\sum_{j=1}^{m} \frac{C B_{j}^{2}}{m x}$

$E S S=\sum_{i=1}^{m^{2}} \sum_{j=1}^{m^{2}} Y_{i j}^{2}-\sum_{l=1}^{x} \frac{R_{l}^{2}}{x}-\sum_{p=1}^{x} \frac{C_{p}^{2}}{x}-\sum_{n=1}^{x} \frac{T_{n}^{2}}{x}-\sum_{i=1}^{m} \sum_{p=1}^{m} \frac{S(\alpha)_{q(i)}}{x}-\sum_{j=1}^{m} \sum_{w=1}^{m} \frac{\pi(\beta)_{w(j)}}{x}+\frac{4 G^{2}}{N}$

Error degrees of freedom $=(m-1)(x+2)$

Table 6: ANOVA table for BISSD Model Type III

\begin{tabular}{|l|l|c|l|l|}
\hline Source & Sum of squares & Degree of freedom & Mean Square & F-Ratio \\
\hline Treatments & SST & $x-1$ & $M S T=S S T / d f$ & $M S T / M S E$ \\
\hline Row & SSR & $x-1$ & $M S R=S S R / d f$ & $M S R / M S E$ \\
\hline Column & SSC & $x-1$ & $M S C=S S C / d f$ & $M S C / M S E$ \\
\hline Row-block & SSRB & $m-1$ & $M S R B=S S R B / d f$ & $M S R B / M S E$ \\
\hline Column-block & SSCB & $m-1$ & $M S C B=S S C B / d f$ & $M S C B / M S E$ \\
\hline Square within RB & SSHS & $m(m-1)$ & $M S H S=S S H S / d f$ & $M S H R B / M S E$ \\
\hline Square within CB & SSVS & $m(m-1)$ & $M S V S=S S V S / d f$ & $M S V C B / M S E$ \\
\hline Error & SSE & $(m-1)(x+2)$ & $M S E=S S R B / d f$ & \\
\hline Total & TSS & $m^{2} x-1$ & & \\
\hline
\end{tabular}




\section{Balanced Incomplete Sudoku square designs - Type 1V}

In this model it is assumed that the row effects and the horizontal square effects are nested in the row block effects and the column effects and the vertical square effects are nested in the column block effects.

$$
\begin{gathered}
Y_{i j(l, n, p, q, w)}=\mu+\alpha_{i x}+\beta_{j x}+\tau_{n}+\gamma(\alpha)_{l(i) x}+C(\beta)_{p(j) x}+S(\alpha)_{q(i) x}+\pi(\beta)_{w(j) x}+e_{i j(l n, p, q, w)} \\
\text { where } i, j, l, p, q, w=1,2, \ldots m, n=1,2, \ldots, x
\end{gathered}
$$

$\mu=$ General Mean effect

$\alpha_{i x}=i^{t h}$ Block (row) effect in $x^{\text {th }}$ empty cells,

$\beta_{j x}=j^{\text {th }}$ Block (column) effect in $x^{\text {th }}$ empty cells,

$T_{n}=n^{\text {th }}$ Treatment effect

$\gamma(\alpha)_{l(i) x}=l^{\text {th }}$ Row effect nested in ith row block effect in $x^{\text {th }}$ empty cells,

$C(\beta)_{p(j) x}=p^{\text {th }}$ Column effect nested in $j t h$ column block effect in $x^{\text {th }}$ empty cells,

$S(\alpha)_{q(i) x}=q^{t h}$ Horizontal square effect nested in ith row block effect in $x^{\text {th }}$ empty cells,

$\pi(\beta)_{w(j) x}=w^{t h}$ Vertical square effect nested in $j t h$ column block effect in $x^{t h}$ empty cells,

$e_{i j(l, n, p, q, w)}=$ Error component with mean zero and variance $\sigma^{2}$

$T S S=\sum_{i=1}^{m^{2}} \sum_{j=1}^{m^{2}} Y_{i j}^{2}-\frac{G^{2}}{N}$

$S S T=\sum_{n=1}^{x} \frac{T_{n}^{2}}{x}-\frac{G^{2}}{N}$

$S S R B=\sum_{i=1}^{m} \frac{R B_{i}^{2}}{m x}-\frac{G^{2}}{N}$

$S S C B=\sum_{j=1}^{m} \frac{C B_{i}^{2}}{m x}-\frac{G^{2}}{N}$

$S S R=\sum_{i=1}^{m} \sum_{l=1}^{m} \frac{R_{l(i)}^{2}}{x}-\sum_{i=1}^{m} \frac{R B_{i}^{2}}{m x}$

$S S C=\sum_{j=1}^{m} \sum_{p=1}^{m} \frac{C_{p(j)}^{2}}{x}-\sum_{j=1}^{m} \frac{C B_{i}^{2}}{m x}$

$S S H S=\sum_{i=1}^{m} \sum_{p=1}^{m} \frac{S(\alpha)_{q(i)}}{x}-\sum_{i=1}^{m} \frac{R B_{i}^{2}}{m x}$

$S S V S=\sum_{j=1}^{m} \sum_{w=1}^{m} \frac{\pi(\beta)_{w(j)}}{x}-\sum_{j=1}^{m} \frac{C B_{j}^{2}}{m x}$

$E S S=\sum_{i=1}^{m^{2}} \sum_{j=1}^{m^{2}} Y_{i j}^{2}+\sum_{i=1}^{m} \frac{R B_{i}^{2}}{m x}+\sum_{j=1}^{m} \frac{C B_{i}^{2}}{m x}-\sum_{n=1}^{x} \frac{T_{n}^{2}}{x}-\sum_{i=1}^{m} \sum_{l=1}^{m} \frac{R_{l(i)}^{2}}{x}-\sum_{j=1}^{m} \sum_{p=1}^{m} \frac{C_{p(j)}^{2}}{x}-\sum_{i=1}^{m} \sum_{p=1}^{m} \frac{S(\alpha)_{q(i)}}{x}-\sum_{j=1}^{m} \sum_{w=1}^{m} \frac{\pi(\beta)_{w(j)}}{x}+\frac{2 G^{2}}{N}$

Error degrees of freedom $=(m+1)(x-1)$. 
Table 7: ANOVA table for BISSD Model Type IV

\begin{tabular}{|l|l|c|c|c|}
\hline Source & Sum of squares & Degree of freedom & Mean Square & F-Ratio \\
\hline Treatments & SST & $x-1$ & $M S T=S S T / d f$ & $M S T / M S E$ \\
\hline Row blocks & SSRB & $x-1$ & $M S R=S S R / d f$ & $M S R / M S E$ \\
\hline Column blocks & SSCB & $x-1$ & $M S C=S S C / d f$ & $M S C / M S E$ \\
\hline Row within RB & SSR & $m(m-1)$ & $M S R B=S S R B / d f$ & $M S R B / M S E$ \\
\hline Column within CB & SSC & $m(m-1)$ & $M S C B=S S C B / d f$ & $M S C B / M S E$ \\
\hline Square within RB & SSHS & $m(m-1)$ & $M S H S=S S H S / d f$ & $M S H R B / M S E$ \\
\hline Square within CB & SSVS & $m(m-1)$ & $M S V S=S S V S / d f$ & $M S V C B / M S E$ \\
\hline Error & SSE & $(m+1)(x-1)$ & $M S E=S S R B / d f$ & \\
\hline Total & TSS & $m^{2} x-1$ & & \\
\hline
\end{tabular}

\section{Illustration.}

Table 8 give hypothetical data of a balanced incomplete Sudoku square design. The BISSD ANOVA tables obtained using R software for models 1 to 4 are presented in tables 9 to 12 , respectively.

Table 8: Hypothetical data of a BISSD

\begin{tabular}{|l|l|l|l|l|l|l|l|l|}
\hline & 35 & 35 & 39 & & 28 & 31 & 42 & \\
\hline 28 & & 26 & 27 & 33 & & & 31 & 37 \\
\hline 24 & 25 & & & 29 & 43 & 41 & & 35 \\
\hline & 31 & 29 & 36 & & 44 & 34 & 27 & \\
\hline 23 & & 35 & 24 & 27 & & & 28 & 43 \\
\hline 28 & 40 & & & 30 & 25 & 36 & & 37 \\
\hline & 33 & 37 & 24 & & 35 & 37 & 40 & \\
\hline 25 & & 26 & 27 & 23 & & & 30 & 25 \\
\hline 27 & 32 & & & 34 & 39 & 28 & & 32 \\
\hline
\end{tabular}

TABLE 9. ANOVA RESULT FOR BISSD MODEL I

\begin{tabular}{|c|c|c|c|c|c|}
\hline $\begin{array}{ll}\text { Source } & s\end{array}$ & Sum of square & degree of freedom & Mean squares & F-Ratio & $\begin{array}{c}\text { Table-value* } \\
5 \% \text { level }\end{array}$ \\
\hline Treatments & 89.7037 & 5 & 17.9407 & 0.7957 & 2.71 \\
\hline Rows & 362.4815 & 8 & 45.3102 & 2.0097 & 2.45 \\
\hline Columns & 484.8148 & 8 & 60.6019 & 2.6879 & 2.45 \\
\hline Row blocks & 35.1481 & 2 & 17.5741 & 0.7795 & 3.49 \\
\hline Column blocks & 159.5926 & 2 & 79.7963 & 3.5392 & 3.49 \\
\hline Sub-square & 252.1481 & 8 & 31.5185 & 1.39792 .45 & \\
\hline Error & 450.9260 & 20 & 22.5463 & — & $\ldots$ \\
\hline Total & 1834.8148 & 53 & & & \\
\hline
\end{tabular}


TABLE 10: ANOVA RESULT FOR BISSD MODEL II

\begin{tabular}{lccccc}
\hline Source & $\begin{array}{c}\text { Sum of } \\
\text { square }\end{array}$ & $\begin{array}{c}\text { degree of } \\
\text { freedom }\end{array}$ & $\begin{array}{c}\text { Mean } \\
\text { squares }\end{array}$ & F-Ratio & $\begin{array}{c}\text { Table-value* } \\
\text { 5\% level }\end{array}$ \\
\hline Treatments & 89.7037 & 5 & 17.9407 & 0.6676 & 2.62 \\
Row blocks & 35.1481 & 2 & 17.5741 & 0.6539 & 3.40 \\
Column blocks & 159.5926 & 2 & 79.7963 & 2.9692 & 3.40 \\
Rows within RB & 327.3334 & 6 & 54.5556 & 2.0230 & 2.51 \\
Columns within CB & 325.2222 & 6 & 54.2037 & 2.0169 & 2.51 \\
Sub-square & 252.1481 & 8 & 31.5185 & 1.1728 & 2.36 \\
Error & 645.0000 & 24 & 26.8750 & - & - \\
Total & 1834.8148 & 53 & - & - & \\
\hline
\end{tabular}

TABLE 11. ANOVA RESULT FOR BISSD MODEL III

\begin{tabular}{lccccc}
\hline Source & $\begin{array}{l}\text { Sum of } \\
\text { Square }\end{array}$ & $\begin{array}{c}\text { degree of } \\
\text { freedom }\end{array}$ & $\begin{array}{c}\text { Mean } \\
\text { squares }\end{array}$ & F-Ratio & $\begin{array}{c}\text { Table-value* } \\
\text { 5\% level }\end{array}$ \\
\hline Treatments & 89.7037 & 5 & 17.9407 & 0.7294 & 2.85 \\
Rows & 362.4815 & 8 & 45.3102 & 1.8423 & 2.59 \\
Columns & 484.8148 & 8 & 60.6019 & 2.4640 & 2.59 \\
Row blocks & 35.1481 & 2 & 17.5741 & 0.7145 & 3.63 \\
Column blocks & 159.5926 & 2 & 79.7963 & 3.2444 & 3.63 \\
Squares within RB & 217.0000 & 6 & 36.1667 & 1.4705 & 2.74 \\
Squares within CB & 92.5555 & 6 & 15.4259 & 0.6272 & 2.74 \\
Error & 393.5186 & 16 & 24.5949 & - & - \\
Total & 1834.8148 & 53 & - & - & \\
\hline
\end{tabular}

TABLE 12: ANOVA RESULT FOR BISSD MODEL IV

\begin{tabular}{|c|c|c|c|c|c|}
\hline$\overline{\text { Source }}$ & $\begin{array}{l}\text { Sum of } \\
\text { square }\end{array}$ & $\begin{array}{r}\text { degree of } \\
\text { freedom }\end{array}$ & $\begin{array}{l}\text { Mean } \\
\text { squares }\end{array}$ & F-Ratio & $\begin{array}{c}\text { Table-value* } \\
5 \% \text { level }\end{array}$ \\
\hline Treatments & 89.7037 & 5 & 17.9407 & 0.6070 & 2.71 \\
\hline Row blocks & 35.1481 & 2 & 17.5741 & 0.5975 & 3.49 \\
\hline Column blocks & 159.5926 & 2 & 79.7963 & 2.7130 & 3.49 \\
\hline Rows within BR & 327.3334 & 6 & 54.5556 & 1.8548 & 2.60 \\
\hline Columns within BC & 325.2222 & 6 & 54.2037 & 1.8428 & 2.60 \\
\hline Squares within $\mathrm{RB}$ & 217.0000 & 6 & 36.1667 & 1.2296 & 2.60 \\
\hline Squares within $\mathrm{CB}$ & 92.5555 & 6 & 15.4259 & 0.5245 & 2.60 \\
\hline Error & 588.2593 & 20 & 29.4130 & 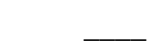 & 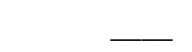 \\
\hline Total & 1834.8148 & 53 & & & \\
\hline
\end{tabular}




\section{CONCLUSION}

In this paper we introduce a new class of design called balanced incomplete Sudoku squares (BISS) design. This design allows experimenter to investigate the blocks and treatments variables, where the size of blocks may be less than the number of treatments. A general construction method of (BISS) design is proposed via orthogonal Sudoku squares. In addition, the models of the orthogonal Sudoku designs where modified to contain balanced incomplete Sudoku squares design.

\section{REFERENCES}

Bejar R., Fernandez C. Mateu C. Megda Valls M. (2012). The Sudoku Completion Problem With Rectangular Hole

Pattern Is NP-Complete. Discrete Mathematics 312, 3306-3315.

Bose R.C., Shrikhande, S.S., Parker, E.T., (1960). Further results on the construction of mutually orthogonal Latin

squares and the falsify of Euler's conjecture. Canadian Journal of Mathematics 12, 189-203.

Colbourn C., The complexity of completing partial latin squares, Discrete Appl. Math. 8 (1984), 151-158.

Danbaba, A., Odeyale. A.B., Musa. Y., (2018). Joint Analysis of Several Experiments Conducted via Orthogonal

Sudoku Design of Odd Order, International Journal of Statistics and Applications, 8(6), 323-331

Das, A., Dey, A. (1990). A note on construction of Graeco Latin square of order $2 n+1$,Journal of India Soc Agric Statist 42, 247-249.D.,
Donovan, D., Haaland B. and Nott D.J (2015). A Simple Approach to Constructing Quasi-Sudoku-based Sliced SpaceFilling Designs. arxiv.1502.05522v1

Hui-Dong M. and Ru-Gen, X. (2008). Sudoku Square- a New Design in field Experiment, Acta Agron Sin, 34(9), 1489-1493.

Kanaana, I. and Ravikumar B. (2010). Row-filled completion problem for Sudoku. Util. Math., 81, 65-84

Kumar, A., Varghese C. Varghese, E. and Jaggi S. (2015). On the construction of designs with three-way blocking. Model;Assisted Statistics and Applications 10, 43-52 43

Mahdian, M., E.S. Mahmoodian (2015) , Sudoku Rectangle Completion, Electronic Notes in Discrete Mathematics 49, 747-755

Mingyao, Ai., Kang, Li., Sanmao, Liu., Dennis, and KJ Lin., (2013). Balanced Incomplete Latin Square Designs. Journal of Statistical and Inference 143 (2013) 1575-1582.

Subramani, J. and K.N. Ponnuswamy (2009). Construction and Analysis of Sudoku designs. Model Assisted Statistics and Applications, 4(4), 287-301.

Subramani, J. (2013). Construction of Graeco Sudoku square Designs of Odd Orders, Bonfring International Journal of Data Mining, 2 (2), 37-41.

Wu C.F.J, and Hamada, M. (2000). Experiments: Planning, Analysis, and Parameter Design Optimization. Wiley, New York. 